\title{
Tevatron Searches for Physics Beyond the SM and the MSSM
}

\author{
David Stuart \\ University of California, Santa Barbara \\ Dept. of Physics, Santa Barbara, CA. U.S.A
}

\begin{abstract}
A survey is given of recent results from searches at the Tevatron for physics beyond the standard model concentrating specifically on searches beyond the traditional supersymmetry signatures.
\end{abstract}

\section{Introduction}

The Tevatron experiments, CDF and D0, have many new results [1] on direct searches for evidence of non-Standard Model phenomena with Run 2 data. Most of these results are obtained from approximately $1 \mathrm{fb}^{-1}$ of integrated luminosity.

Searches can either be structured to have optimal sensitivity to specific models, or they can be optimized for broader sensitivity by looking for deviations in specific final states. Searches that focus on the minimal super-symmetric standard model are examples of the former; the results of those searches are given elsewhere [2] in these proceedings. In this talk I cover non-SUSY searches and separate them into model specific and signature specific searches.

The slides [3] contain plots of the data and results. Reproducing those plots here is not efficient. Instead, I concentrate on discussion of the results.

\section{$2 \quad$ Model specific searches}

Perhaps the most familiar non-SUSY search modes are for new massive gauge bosons, which appear in many unification models. A new neutral gauge boson, a $Z^{\prime}$, would manifest as a narrow di-lepton mass bump. The most recent such search from CDF uses di-electrons. The main background is high mass Drell-Yan production. Instrumental backgrounds, such as di-jets faking electrons, are quite small by comparison. The mass region above $150 \mathrm{GeV} / \mathrm{c}^{2}$ is scanned for resonances; there are some bins that fluctuate, as is expected with many trials, but none are significant. Cross-section limits of about $10 \mathrm{fb}$ are derived for $Z^{\prime}$ masses above the highest mass events observed, which are at about $500 \mathrm{GeV} / \mathrm{c}^{2}$. This corresponds to a mass limit of $920 \mathrm{GeV} / \mathrm{c}^{2}$ assuming SM like couplings.

Similarly, D0 has completed a recent search for a new charged boson, a $W^{\prime}$ in the $e \nu$ channel. A $W^{\prime}$ would produce a broad excess at large transverse mass, where the dominant background is the high mass tail of $W \rightarrow e \nu$. Instrumental backgrounds are again negligible. The data is well described by the background prediction, with the highest transverse mass event having $620 \mathrm{GeV} / \mathrm{c}^{2}$. Cross-section limits of about $20 \mathrm{fb}$ are obtained, which correspond to a mass limit of $965 \mathrm{GeV} / \mathrm{c}^{2}$ assuming SM like couplings.

Searches for extra-dimension models have produced recent results in both RS [4] and ADD [5] models. CDF has searched for resonant $G \rightarrow \gamma \gamma$ production, which they combine with $G \rightarrow e e$ results derived from the di-electron search described above. D0 directly combines the $e e$ and $\gamma \gamma$ modes at the analysis stage by not requiring a track match for their 
electron candidates. Both experiments present mass limits on RS gravitons as a function of the coupling, $k / M_{\mathrm{pl}}$, that range from about $300 \mathrm{GeV} / \mathrm{c}^{2}$ at $k / M_{\mathrm{pl}}=0.01$ to $860 \mathrm{GeV} / \mathrm{c}^{2}$ for $k / M_{\mathrm{pl}}=0.1$.

Another RS mode is $G \rightarrow Z Z$, which CDF searched using the four electron final state. The background is very small for this mode; the SM ZZ contribution at high mass is negligible, and the $\mathrm{Z}$ plus jet-fakes contribution is small. However, the expected signal is also quite small. The graviton branching ratio to $\mathrm{ZZ}$ is higher than to $e e$ or $\gamma \gamma$, but the $Z \rightarrow e e$ branching ratio penalty is large and comes in squared. So, they used very loose electron selection and a $\chi^{2}$ measure of the $Z$ mass consistency. They observe no high mass events, but do find one low mass event that is consistent with the 0.5 events expected from SM $Z Z$.

The searches for RS models involve resonant graviton production. A difference class of models, the ADD models with large extra dimensions, involve production of a Kaluza-Klein tower of gravitons that radiate into the bulk and cause missing energy. Final state graviton radiation, i.e., $g g \rightarrow g \rightarrow g G$, would produce a monojet plus missing transverse energy (MET). CDF has searched for that signature, in fact allowing one or two jets for better sensitivity. There are backgrounds from QCD with fake MET, $W$ 's, and $Z \rightarrow \nu \nu+$ jet. The jet $E_{T}$ spectrum from a graviton signal does not differ substantially in shape from $Z \rightarrow \nu \nu+$ jet. It is just a tower of many closely spaced gravitons that would produce a falling $E_{T}$ spectrum similar to the QCD radiation in $Z+$ jet. As such, the search amounts to checking the normalization, not the shape. Monte Carlo predictions are not sufficiently reliable for that, so data is used to estimate all the backgrounds, e.g., $Z \rightarrow e e+$ jet is used to predict $Z \rightarrow \nu \nu+$ jet with $\approx 10 \%$ uncertainty. With careful selection, the QCD background becomes negligible and the irreducible $Z$ background dominates. The missing energy spectrum in data is consistent with the background estimate, and limits are derived corresponding to effective planck masses of about $1 \mathrm{TeV}$, with some variation depending on the number of large extra dimensions.

D0 has recently completed a search for an excited electron, which would be evidence for compositeness. The production could occur through a contact interaction, and the signature is a narrow resonance in the $e \gamma$ spectrum, with the normalization dependent on the compositeness scale. As in the previously discussed searches, the QCD background is negligible compared to the irreducible background, in this case from Drell-Yan $+\gamma$ production. D0's data is in good agreement with the expected background, and they derive limits on the compositeness scale ranging from $1 \mathrm{TeV}$ for $m_{e *}=800 \mathrm{GeV}$ up to $6 \mathrm{TeV}$ for a low mass excited electron. Similar analyses have been done searching for excited muons [6].

D0 has a recent result from a search for a second generation leptoquark. Leptoquarks are bosons that carry both color and lepton number and arise in models attempting to unify the lepton and quark families. In the case that one of the, pair-produced, LQs decays to a muon and the other to a neutrino, the signature is a muon, 2 jets, and MET. The backgrounds from $W+$ jets and top are large, but LQs give a characteristic kinematic signature in that the muon and one of the jets would reconstruct to a peak at the LQ mass. D0's data is consistent with the expected background, and cross section limits of about $100 \mathrm{fb}$ are obtained that correspond to masses of about $210 \mathrm{GeV}$.

$\mathrm{CDF}$ has completed a search for a 4 th generation quark, a b prime, decaying to a $\mathrm{Z}$. Since a 4th generation quark is constrained to have small couplings to the 1st three generations, the direct decay to $W$ plus charm is suppressed and it could decay via a loop diagram to a $Z$ and a $b$. They select events with a dilepton $Z$ plus at least 3 jets and then look for large total jet energy that would come from a massive object. The dominant background is SM 
$Z+$ jets, which they predict using the data because this is a regime that is not expected to be well-modeled by LO or NLO MCs. That data-based prediction is made by fitting the jet $E_{T}$ spectrum below the $30 \mathrm{GeV}$ jet threshold and integrating the projection above the threshold to obtain the total number of events with 3 or more jets. Then they fold the predicted jet $E_{T}$ spectra into a sum of jet energy and find the data to be consistent. As an interesting validation check, they use the top sample to check that they can similarly predict the $W+$ multi-jet background solely from low $E_{T}$ data. The data's $W+3$ jet excess over that prediction matches the expected top contribution.

\section{$3 \quad$ Signature specific searches}

The previously described $b^{\prime}$ search did not use a $b$-tag even though the $b^{\prime} \rightarrow b Z$ model would have two $b$ jets. Requiring a $b$-tag would likely have improved the sensitivity to that particular model, but it would make the search insensitive to models without $b$ quarks. That is an example of the trade-off between optimizing for sensitivity to a specific model or to a broader range of new phenomena, and that search was indeed intended to be sensitive to more than just a $b^{\prime}$. I'll now describe several other Tevatron searches that aim to be model-independent, that is, they trade model-specific sensitivity for breadth of sensitivity.

In addition to the $Z+\geq 3$ jet search, CDF has carried out a general search for high $p_{T}$ $Z$ 's. Any massive particle that decays to a $Z$ would give it high $p_{T}$, mostly independent of the details in the model. The $Z p_{T}$ spectrum in data is observed to be consistent with background expectations from Monte Carlo, but the background is large. To expand sensitivity, they search for other objects in addition to the $Z$; additional leptons, photons, MET, or large total energy $\left(H_{T}\right)$. These modes encompass several SM background sources, such as $W Z, Z \gamma$, and $Z Z$. The goal is sensitivity to a breadth of new physics, but it is also sensitive to how well one understands many different SM processes. There is fair agreement. For example, 14 events are found in both the $e e \gamma$ and $\mu \mu \gamma$ category, with expected backgrounds of $12.4 \pm 1.5$ and $15.0 \pm 1.8$ events, respectively. The $e e+H_{T}$ and $\mu \mu+H_{T}$ categories contain 45 and 53 events with expected backgrounds of $36.4_{-5.8}^{+4.9}$ and $41.3_{-5.2}^{+5.0}$ events, respectively. The $e e+\mathrm{MET}$ and $\mu \mu+\mathrm{MET}$ categories contain 97 and 74 events with expected backgrounds of $85.4 \pm 12.3$ and $55.9_{-5.8}^{+9.7}$ events, respectively.

This approach has been generalized from $Z$ specific dileptons to all dileptons, which expands the range of SM background sources to include $W W$ and top. They again search for additional objects in the event adding high $E_{T}$ and $b$-tagged jets to the list given above. This search finds one $e \mu$ event with a total energy of over $850 \mathrm{GeV}$ with a $b$-tag and 3 jets, which looks top-like upon close inspection.

A similar CDF search examines di-photon events looking for other objects: leptons, MET, or another photon. There was, of course, one event in Run 1 with two photons, two electron candidates and missing energy that would fall with this search. They find no anomalies in the $\gamma \gamma+$ lepton category with 3 events observed and $7.6 \pm 0.8$ expected. Four events are observed with three photons compared to an expected background of $2.2 \pm 0.7$. One di-photon event with MET> $75 \mathrm{GeV}$ is found with $0.24 \pm 0.22$ expected.

The final result presented is a search for a stable, charged, massive particle. The search is designed to be model independent, although example model motivations come from a stau or stop as the next to lightest susy particle with long lifetime due to suppressed couplings in gauge mediated susy breaking scenarios. A long-lived gluino in split-susy is another, more recently popular, scenario. Any such heavy charged particle would penetrate the calorimeters 
like a muon because there is insufficient energy in the center of mass for showering to occur when the calorimeter's nuclei collide with the particle. But, it would be slow; a slow muon. D0 previously carried out a search looking for late hits in the muon chamber scintillators. CDF has recently completed a search using its time of flight counters, which have resolution of order $100 \mathrm{ps}$. Given the speed, $\beta$, obtained from the timing, and the momentum, the particle's mass is calculated. They use electrons from $W$ 's as a control sample for the timing resolution and obtain a background prediction from lower $p_{T}$ muons. No excess is found in the high $p_{T}$ and high mass muon sample. Model independent limits are calculated, without unfolding acceptance, of about $10 \mathrm{fb}$ for weakly interacting particles. The limit for strongly interacting particles is about 5 times larger due to fragmentation effects.

\section{Summary}

There are several general things to note about this broad survey of results. In most of the analyses, instrumental backgrounds such as QCD jets faking leptons are negligible. This was not the case in early Tevatron results; it is apparent that the experiments have gained a mature understanding of their data and methods. Several of the analyses use data-based background prediction methods to probe regions beyond the normal purview of LO or NLO Monte Carlo calculations.

No significant excesses were observed in these analyses. Several $\approx 2 \sigma$ fluctuations are present, as there should be after examining many different modes and a large number of kinematic bins. Those deviations that are merely statistical fluctuations will disappear in the six to eight fold larger final Tevatron sample (and be replaced by other fluctuations). Deviations that are real, but not yet significant, would grow, which makes the future of Tevatron search results interesting.

\section{References}

[1] All CDF and D0 results are available at http://www-cdf.fnal.gov and http://www-do.fnal.gov

[2] R. Strohmer, these proceedings.

[3] Slides: http://indico.cern. ch/contributionDisplay.py? contribId=132\&sessionId=9\&conf Id=9499

[4] L. Randall and R. Sundrum, Phys. Rev. Lett. 833370 (1999); Ibid 4690.

[5] N. Arkani-Hamed, S. Dimopoulos, G. Dvali, Phys. Lett. B429 263 (1998).

[6] A. Abulencia et al., The CDF Collaboration, Phys. Rev. Lett. 97191802 (2006); V.M. Abazov et al., The D0 Collaboration, Phys. Rev. D 73111102 (2006) 